\title{
Compact Objects in Supernova Remnants
}

\author{
By ROGER A. CHEVALIER
}

\author{
University of Virginia, Charlottesville, VA 22903-3818, USA
}

\begin{abstract}
Core collapse in very massive stars can lead to a central black hole that swallows the rest of the star and in less massive stars to a central neutron star and explosion. There is probably an intermediate mass range that gives an explosion and a central black hole; supernova remnants with no observable central object are candidates. The association of pulsars with Type II supernovae gives an estimate of the pulsar power to be expected in a supernova, but the uncertainty in the initial pulsar periods gives a wide range in possible powers. The relativistic wind bubble model for the Crab Nebula has steadily developed and there are now predictions regarding particle acceleration in the optical wisps. The bubble model with expansion into supernova gas can also be applied to other young pulsar nebulae.
\end{abstract}

\section{Introduction}

The study of compact objects in supernova remnants has long been troubled by the lack of evidence for such objects. For many years, the Crab and Vela pulsars were the only compact objects observed in remnants. More recently, the number of pulsar/remnant associations has increased to 9 or 10 (Kaspi et al. 1992; Kulkarni et al. 1993). In other cases, the presence of a pulsar is inferred from a centrally condensed, flat radio spectrum nebula thought to be created by a pulsar. The study of these objects, as well as more detailed study of the Crab Nebula, has led to a general theoretical picture, although many basic uncertainties remain.

In section 2, I describe the possibilities for forming neutron stars or black holes in core collapse supernovae. Section 3 discusses the implications of observed pulsars for supernovae. The Crab Nebula and its evolution are described in section 4 and recent investigations of other pulsar nebulae are described in section 5 .

\section{The Nature of the Compact Objects}

Core collapse is thought to occur at the end of the lives of stars with an initial mass $z 8 M_{\odot}$, although there may be a high mass range $\left(z 100 M_{\odot}\right)$ where oxygen burning can lead to the complete destruction of the star. The Fe core mass ranges from $\sim 1.4 M_{\odot}$ for the $\lesssim 18 M_{\odot}$ star to $\sim 2 M_{\odot}$ for the more massive stars (S. Woosley, this volume); the core mass is not necessarily a monotonic function of initial mass. The maximum mass of a neutron star, $M_{c r}$, is not well-determined, but probably lies in the range 1.5$2.0 \mathrm{M}_{\odot}$. Thus, in the more massive stars, core collapse can lead directly to black hole formation. The generation of a supernova shock by any plausible mechanism requires the initial formation of a neutron star, so the star remains bound to the black hole and is accreted by it. This is thought to be the origin of massive black holes like Cygnus X-1.

The timescale to launch the supernova shock may be $\sim 1$ second. There are two ways to form a black hole after this time. One is by the fall-back of matter that was initially ejected by the supernova shock. Colgate (1971) and Chevalier (1989) discussed how this could happen as a result of pressure waves or reverse shock waves during the initial expansion of the supernova. Chevalier $(1989,1991)$ estimated that $0.1 M_{\odot}$ could fall back on a timescale of hours after the explosion. Brown, Bruenn, \& Wheeler (1992) 
estimated a similar amount of fall-back (up to $1 M_{\odot}$ ) during the hot bubble phase on a timescale of $10^{3}$ seconds. They also estimated that $M_{c r}$ is fairly low, about $1.5 M_{\odot}$. If these estimates are reliable, there should be an initial core mass range just below $M_{c r}$ where the fall-back can give a black hole with an explosion. We do not know the relevant parameters sufficiently well to estimate the initial mass range.

Another way to achieve a black hole with explosion is to form a hot neutron star that can remain stable above $M_{c r}$ that then cools on a $>1$ second timescale and forms a black hole (e.g. Brown \& Bethe 1993). Again, the mass range is very uncertain, although Brown and Bethe suggest that it is large.

If a black hole does form at the center, the compact object is likely to be difficult to observe. Chevalier (1989) estimated that the late fall-back rate for SN 1987A was $\dot{M} \sim 10^{-4} t_{\mathrm{yr}}^{-5 / 3} M_{\odot} \mathrm{yr}^{-1}$, where $t_{\mathrm{yr}}$ is the age in years. The $t^{-5 / 3}$ factor is generally expected for late fall back (see also Michel 1988), but the coefficient may vary with the details of the supernova. In spherically symmetric accretion, the radiation field becomes trapped in the inflow so that the only luminosity is due to compression outside the radiation trapping radius. The resulting luminosity is low, about $5 \times 10^{35} t_{\mathrm{yr}}^{-1.4} \mathrm{ergs} \mathrm{s}^{-1}$ for the above accretion rate. If angular momentum of the material causes a disk to form, the radiative efficiency may be considerably higher.

The possible low radiative output of black holes has led to the suggestion that they are present in supernova remnants that should have a central compact object but show no sign of one, such as SN 1987A and Cas A. In the case of SN 1987A, the observed neutrino burst directly implies that a neutron star or black hole is present, but the luminosity from a pulsar was $\lesssim 8 \times 10^{36} \mathrm{ergs} \mathrm{s}^{-1}$ on day 1500 (Suntzeff et al. 1992). However, a neutron star with a low power output could be present. The minimum luminosity is thermal emission from the hot neutron star surface soon after formation. At an age of 1 year, the expected temperature of $3 \times 10^{6} \mathrm{~K}$ gives a luminosity of $\sim 10^{35} \mathrm{ergs} \mathrm{s}^{-1}$ (Nomoto \& Tsuruta 1987). Unfortunately, in the case of SN1987A, the longer lived radioactive nuclides, such as ${ }^{44} \mathrm{Ti}$, can dominate this emission for decades (Woosley, Hartmann \& Pinto 1989). In the case of Cas A, thermal emission from the hot gas impedes the detection of a central soft $\mathrm{X}$-ray source.

\section{Pulsars and Supernovae}

Observations of supernovae during the first few years after the explosion have not given any information on the nature of central compact objects. However, it has long been held that there is a correspondence between Type II supernovae and pulsar formation so that an analysis of pulsar properties can tell us what to expect inside of these supernovae. There are basically three reasons supporting this correspondence. First, the rate of pulsar formation in our Galaxy is comparable to the Type II supernovae rate, both are about 1 per 50 years, within a factor of 2 (Gunn \& Ostriker 1970; Narayan \& Ostriker 1990; van den Bergh \& Tammann 1991). The second is that although the pulsar dispersion in the $z$ direction (galactic altitude) is large, the dispersion can be attributed to the high space velocity of pulsars and the data are consistent with pulsar birth in a narrow region close to the galactic plane, as expected for a massive star origin (Gunn \& Ostriker 1970; Chevalier \& Emmering 1986). However, Narayan \& Ostriker (1990) have claimed that there are two populations of pulsars with roughly equal birthrates, one born with a large scale height $(z 350 \mathrm{pc})$ and the other born with a small scale height $(\sim 120 \mathrm{pc})$. This claim needs further confirmation, but it is certainly possible that Type II supernovae should be identified with a subset of the pulsars. Finally, the association of the Crab 
TABLE 1. Observed Pulsars in Nebulae

\begin{tabular}{cccc}
\hline & PSR 0531+21 & PSR 0540-69 & PSR 1509-58 \\
\hline$P(\mathrm{~s})$ & 0.0333 & 0.0503 & 0.150 \\
$\dot{P}$ & $4.21 \times 10^{-13}$ & $4.77 \times 10^{-13}$ & $1.54 \times 10^{-12}$ \\
$n$ & 2.509 & 2.01 & 2.83 \\
$\dot{E}\left(\mathrm{erg} \mathrm{s}^{-1}\right)$ & $4.5 \times 10^{38}$ & $1.5 \times 10^{38}$ & $1.8 \times 10^{37}$ \\
$t+\tau(\mathrm{yr})$ & 1660 & 3310 & 1690 \\
$t(\mathrm{yr})$ & 930 & 830 & 1660 \\
$\tau(\mathrm{yr})$ & 730 & 2480 & 30 \\
$\dot{E}_{i}\left(\mathrm{erg} \mathrm{s} \mathrm{s}^{-1}\right)$ & $3.0 \times 10^{39}$ & $3.5 \times 10^{38}$ & $8.3 \times 10^{40}$ \\
$P_{i}(\mathrm{~s})$ & 0.019 & 0.038 & 0.017 \\
$B_{s}\left(10^{12} \mathrm{G}\right)$ & 3.8 & 5.0 & 15.4 \\
\hline
\end{tabular}

pulsar with SN 1054 has provided a direct link of pulsar formation with a supernova explosion.

The study of pulsars can thus lead to information on the properties of neutron stars to be expected in supernovae. The power output from the neutron star is a fundamental property and in the magnetic dipole theory for pulsar spindown, the power output is

$$
\dot{E}=5 \times 10^{38}\left(\frac{B_{s}}{10^{12.5} \mathrm{G}}\right)^{2}\left(\frac{P}{30 \mathrm{msec}}\right)^{-4} \operatorname{ergs~s}^{-1},
$$

where it has been assumed that the magnetic axis is perpendicular to the rotation axis, $B_{s}$ is the surface magnetic field and $P$ is the pulsar spin period. Analyses of pulsar statistics have led to estimates of the initial values of $B_{s}$ and $P$ for pulsars. There is general agreement that the initial values of $B_{s}$ fall in a fairly narrow range $-10^{12.5 \pm 0.3} \mathrm{G}$ (Gunn \& Ostriker 1970; Stollman 1987) and any magnetic decay occurs on a timescale that is longer than the ages of supernova remnants. An increase in $B_{s}$ on a timescale smaller than the typical pulsar ages is possible (Blandford, Applegate, \& Hernquist 1983). The initial periods of pulsars have not been well-determined from pulsar statistics because of the small number of young, rapidly spinning pulsars and uncertainties in the selection effects. Initial periods for most pulsars from $\sim 10 \mathrm{msec}$ to $\sim 0.7 \mathrm{sec}$ have been suggested (Chevalier \& Emmering 1986; Narayan 1987; Stollman 1987; Emmering \& Chevalier 1989; Narayan \& Ostriker 1990). The difference in initial power output is large: for $B_{s}=10^{12.5} \mathrm{G}$, the power of a $10 \mathrm{msec}$ period pulsar is $4 \times 10^{40} \mathrm{ergs} \mathrm{s}^{-1}$, while that of a $0.7 \mathrm{sec}$ period pulsar is $1.6 \times 10^{33} \mathrm{ergs} \mathrm{s}^{-1}$.

Some information can be obtained from the 3 youngest known pulsars, which have ages of $\sim 10^{3}$ years. The basic data on these pulsars is given in Table 1 (for references to the observations, see Chevalier \& Fransson 1992), which includes the period $P$, first period derivative $\dot{P}$, and braking index $n=\Omega \ddot{\Omega} / \dot{\Omega}^{2}$, where $\Omega=2 \pi / P$ is the spin rate. The current spin-down power is $\dot{E}=I \Omega \dot{\Omega}$, where $I$ is the neutron star moment of inertia and is taken to be $10^{45} \mathrm{~g} \mathrm{~cm}^{2}$ in Table 1 . If the pulsar spin evolves with constant braking index $n$, the initial spin rate is $\Omega_{i}=\Omega(1+t / \tau)^{1 /(n-1)}$, where $\tau$ is a constant and $t$ is the age. We also have that $P / 2 \dot{P}=(\tau+t)(n-1) / 2$. The age of the Crab pulsar is known and that of $0540-69$ can be deduced from the expansion age of the nebula (Kirshner et al. 1989), with some correction for acceleration of the nebula (Reynolds 1985; Chevalier \& Fransson 1992). The age of $1509-58$ is determined if it was born in SN 185 (Thorsett 
1992) but the values of $t$ and $\tau$ given here are based on a speculative model described in section 5. The result of an initial period for 1509-58 comparable to that of the Crab pulsar is thus speculative. The high initial power of this pulsar is due to its unusually high magnetic field.

These results use the observed braking index, which, in every case, is less than the value expected for pure magnetic dipole spindown, $n=3$. The lower value may be due to a particle wind from the neutron star. The use of $n=3$ to extrapolate back to the initial values would not greatly change the results. In the magnetic dipole model, $\tau=$ $3 I c^{3} / 4 B_{s}^{2} R_{N}^{6} \Omega_{i}^{2}$, where $c$ is the speed of light and $R_{N}$ is the neutron star radius.

These three pulsars lead to the best estimates for the original periods of individual pulsars. It is not clear whether their fairly fast initial spin rates are representative because there is a selection effect to observe the brightest pulsars and pulsar nebulae. Srinivasan, Bhattacharya, \& Dwarakanath (1984) estimated the galactic rate of formation of pulsar nebulae like the Crab Nebula to be 1 per 240 years, only 20\% of the estimated Type II supernova or pulsar formation rate. From radio observations of the galaxy M33, Reynolds \& Fix (1987) deduced a similarly low rate of Crab-type nebula formation in that galaxy as an upper limit. Srinivasan et al. concluded that most Galactic pulsars are probably formed with $P_{i} \gtrsim 35-70 \mathrm{msec}$.

Another piece of evidence for a long initial period is the general lack of observed pulsars in shell supernova remnants. Although this problem is influenced by selection effects, Narayan \& Schaudt (1988) found that the implication is either that there are no pulsars in these supernova remnants or that the pulsars are unusually weak. A solution to the problem is that pulsars are born with periods $\gtrsim 0.7 \mathrm{sec}$. However, one uncertainty is the beaming function for young pulsars. Narayan and Schaudt argue that young pulsars have fan radio beams and are thus always observable, but this result remains controversial (Frail \& Moffett 1993). Also, at the time of the Narayan \& Schaudt paper, there were 4 pulsar/supernova remnant associations, but that number has now grown to 9 or 10 .

Chevalier \& Fransson (1992) addressed the question if a Crab-like pulsar does form in a Type II supernova, what are the effects on the supernova emission during the first decade after the explosion. The magnetic field and relativistic particles powered by the spin-down of the pulsar create a bubble that drives a shock front into the supernova gas. The shock front is initially radiative and the radiative luminosity is $\sim 1.5 \%$ of $\dot{E}$. A higher radiative efficiency ( $30 \%$ in the case of the $\mathrm{Crab}$ ) is possible if the pulsar bubble is a significant source of synchrotron emission; there is evidence that pulsars with higher values of $\dot{E}$ have nebulae with higher efficiencies of synchrotron emission (Seward \& Wang 1988). The ionizing radiation can create an ionized region from which optical lines like those of [OIII] are prominent; these lines are from more highly ionized atoms than those expected from radioactive power input. The lines tend to start out narrow and broaden with time as more of the supernova matter is ionized. Chevalier \& Fransson (1992) examined observations of supernovae over the age range up to 10 years and found that none of them clearly show evidence for pulsar-powered emission; the supernovae in question are generally radio emitters and have strong circumstellar interaction that powers the observed luminosity. The continued observation of Type II supernovae to faint luminosity levels is important for the discovery of very young pulsars. In the meantime, we can examine the properties of pulsars and their nebulae with ages $\sim 10^{3}$ years.

\section{The Crab Nebula and its Evolution}

The observed kinetic energy of the Crab Nebula is only $\sim 3 \times 10^{49}$ ergs, which is far short of the $10^{51}$ ergs typically thought to be associated with Type II supernovae. 
The problem is aggravated by the evidence for acceleration of the Crab Nebula filaments (Trimble 1968), which suggests that some of the kinetic energy has been derived from the pulsar spin-down power. Partly for this reason, Chevalier (1977) and Chevalier \& Fransson (1992) proposed that the observed Crab Nebula is just the inner part of a typical Type II supernova that has been swept up by the pulsar bubble. If a pulsar with the properties described in Table 1 for PSR $0531+21$ is placed inside of a typical Type II supernova, the average shell velocity $\left(1400 \mathrm{~km} \mathrm{~s}^{-1}\right)$, shell mass $\left(1-2 M_{\odot}\right)$, acceleration $(t-R / V \sim 100$ years $)$, and bubble pressure $\left(\sim 10^{-8}\right.$ dynes $\left.\mathrm{cm}^{-2}\right)$ of the Crab Nebula can be roughly reproduced. The filamentary structure of the nebula can be the result of Rayleigh-Taylor instabilities in the acceleration process.

Although this model has a number of attractive features, it does predict that an energetic supernova lies outside the presently observed $\mathrm{Crab}$, and no such object has been clearly observed. The interaction of the rapidly moving gas with the surrounding medium might be most sensitively seen as a shell radio supernova remnant, but no radio shell has been observed to a low level (Velusamy 1985). The implication is that the surrounding density must be low, as might be expected if the main sequence progenitor star cleared a hole with its wind. A low surrounding density implies that much of the supernova gas is in free expansion and is cool. The gas is ionized by the nebular radiation and is expected to be a source of line emission. Murdin \& Clark (1981) did in fact find evidence for a halo around the Crab, but this observation has not yet been confirmed; the problem is the very low emission measure of the expected halo.

Although there is no clear evidence for the interaction of the Crab Nebula with an external medium, there have been recent claims that the bays seen in the synchrotron emission are related to the circumstellar medium around the progenitor star. Fesen, Martin, \& Shull (1992) studied the bays on either side of the pulsar in the east-west direction and found that they are long-lived features that are expanding with the nebula. The bays line up with a band of He-rich filaments that cross the nebula. Fesen et al. (1992) suggest that the filaments came from a disk surrounding the progenitor star outside of which was a magnetic torus. A somewhat different model for the bays was proposed by Li \& Begelman (1992), who suggest that the Crab Nebula is interacting with a slow progenitor wind that is 5 times denser in the equatorial plane than along the poles.

If these circumstellar interaction models have validity, there is a problem with the normal Type II scenario discussed above because the energetic supernova gas would disrupt the nearby circumstellar medium. However, there are possible problems with circumstellar interaction. Images of the outer line emission from the Crab Nebula (e.g. Chevalier \& Gull 1975) show no evidence for an indentation at the position of the bays, as would be expected for current circumstellar interaction with an asymmetric medium. The asymmetric interaction could have occurred at an earlier time. Also, if the filaments lying across the bays had a partial circumstellar origin, they would be expected to have less $\mathrm{He}$ than the average for the Crab Nebula rather than more. The previous explanation for the Crab bays is that they are related to the complex interaction of the pulsar magnetic field with the filaments and this may still be a viable model (cf. Michel et al. 1991).

The standard model for the internal structure of the Crab Nebula is a bubble created by a relativistic, particle dominated wind that is shocked and fills most of the volume (Kennel \& Coroniti 1984a,b and references therein). The shock front is at about $1 / 20$ the nebular radius at the position of optical filaments in the synchrotron emission. In the model of Kennel \& Coroniti, the observed synchrotron emission at optical and higher frequencies can be explained if the Lorentz factor in the wind is $\gamma \sim 10^{6}$. This model predicts a toroidal magnetic field structure and at $\mathrm{X}$-ray wavelengths there is evidence for 
a toroidal structure at the position of suspected wind shock (Aschenbach \& Brinkmann 1975). The position of the structure implies that the rotation axis of the pulsar is aligned in the NW-SE direction (projected against the sky). The outer contours of the Crab Nebula are elongated in the same direction. Begelman \& Li (1992) extended the theory of Kennel and Coroniti to allow for an asymmetry in the nebula. The effect of the tension in the toroidal magnetic field is to reduce the pressure in the equatorial direction, while the polar pressure is not affected. The result is that the wind bubble extends further along the rotation axis, as observed. Begelman \& $\mathrm{Li}$ (1992) found that for the wind magnetization parameter deduced by Kennel and Coroniti, the expected degree of asymmetry in the nebula is close to that observed. A problem with this picture is that the polarization of the optical synchrotron nebula clearly shows a toroidal field structure, but with the axis in the $\mathrm{N}-\mathrm{S}$ direction (Michel et al. 1991). Michel et al. (1991) suggest that the misalignment is related to precession of the pulsar axis. Another possibility is that the magnetic structure is subject to instabilities.

In the Kennel and Coroniti model, particle acceleration occurs at the termination shock of the relativistic pulsar wind, but they did not address the details of the acceleration mechanism. The problem with the usual diffusive shock acceleration mechanism is that it does not operate for a perpendicular shock (magnetic field perpendicular to the shock normal), as expected here. Hoshino et al. (1992) have carried out plasma simulations of a relativistic perpendicular shock to investigate the microphysics of acceleration. A pure electron/positron wind, as is often assumed for pulsars, gives rise to a thermal particle distribution (Gallant et al. 1992), which is not observed. However, if most of the energy flux is in ions, the ions experience reflections at the shock and the magnetic field overshoots its final downstream value, leading to oscillations on the scale of the ion Larmor radius. The ions transfer energy to a nonthermal distribution of positrons and electrons on this scale. For a $\gamma=10^{6}$ wind, the ion Larmor radius is $\sim 0.01 \mathrm{pc}$, or $\sim$ 1 arcsec at the distance of the Crab Nebula. Hoshino et al. identify the wisp structure seen in a high resolution optical image of the Crab Nebula (van den Bergh \& Pritchet 1989) with the acceleration region and predict that the synchrotron spectrum should systematically change with distance from the pulsar. Although the theory does not yet explain the radio synchrotron emission, Bietenholz \& Kronberg (1992) found that the radio spectral index shows changes on a scale comparable to that of the optical wisps, supporting the hypothesis of particle acceleration on this scale.

\section{Other Pulsar Nebulae}

The properties of PSR 0540-69 in the Large Magellanic Cloud are given in Table 1. As with the Crab Nebula, when a pulsar with these properties is placed inside of a normal Type II supernova, the resulting nebula does have characteristics like those of the 0540-69 nebula (Reynolds 1985; Chevalier \& Fransson 1992). A difference with the Crab is that a cooling shock is expected at the edge of the bubble if the gas is enriched in oxygen and the radiative shock power can explain the optical line emission from the nebula. Kirshner et al. (1989) found that the high temperature derived from the [OIII] lines is indicative of shock emission in 0540-69, as opposed to photoionization in the Crab Nebula. Imaging of the nebula in the [OIII] line shows a larger extent than in the continuum or other lines (Caraveo et al. 1992), which would also appear to be consistent with a shock origin. The $\mathrm{H} \alpha$ image of Caraveo et al. (1992) shows a curious hourglass structure, which does not have a straightforward explanation. The model of a pulsar bubble inside of a Type II supernova has been strengthened for $0540-69$ by the detection of a radio shell that has 
similar properties to those of a shell supernova remnant (Manchester, Staveley-Smith, \& Kesteven 1993).

The properties of PSR 1509-58 are also given in Table 1. Chevalier \& Fransson (1992) chose a value of $\tau$ for this pulsar that is quite small for two reasons. First, a small $\tau$ implies that the pulsar had a large initial power, which is needed to create an X-ray synchrotron nebula as large as that observed (Seward et al. 1984). Second, it implies a large value for $t$, which is needed to reduce the average velocity of the MSH 15-52 nebula (Seward et al. 1983) to $\sim 10,600 \mathrm{~km} \mathrm{~s}^{-1}$; this value is high, but is perhaps plausible if the supernova has been expanding into a mass loss wind bubble. More recently, Thorsett (1992) has identified the birth of PSR 1509-58 with SN 185. This implies an age that again requires minimizing the contribution of $\tau$ to the value of $\tau+t$.

Finally, I consider the nebula 3C 58, the likely remnant of SN 1181, which does not have an observed radio pulsar, but is believed to be powered by a pulsar based on the radio morphology and the presence of an X-ray point source (Becker et al. 1982). Frail \& Moffett (1993) have recently undertaken a high spatial resolution radio study of 3C 58 and have found no evidence for a radio pulsar, suggesting that the pulsar is not beamed toward us despite the youth of the object. Frail and Moffett did observe a narrow filament near the X-ray point source and they identify the filament with the toroidal termination shock of the pulsar wind, analogous to the optical wisps near the Crab pulsar. The axis of the torus is along the long axis of the radio synchrotron nebula (Reynolds \& Aller 1988), as expected in the models of asymmetric expansion discussed for the Crab Nebula. The position of the termination shock, together with the pressure in the nebula, gives an estimate of the spin-down power of the pulsar, $\dot{E}=1.5 \times 10^{36} \mathrm{ergs} \mathrm{s}^{-1}$, which approximately agrees with that deduced from the X-ray luminosity of the synchrotron nebula (Seward \& Wang 1988). Frail and Moffett take $P / 2 \dot{P} \approx t$ (i.e. $\tau$ is small and the pulsar is substantially spun down) to derive $P=730 \mathrm{msec}$ and $\dot{P}=15,000 \times 10^{-15} \mathrm{~s} \mathrm{~s}^{-1}$. In the magnetic dipole theory, these values yield the extraordinarily high magnetic field strength of $1 \times 10^{14} \mathrm{G}$. An alternate hypothesis is that $\tau$ makes a major contribution to $P / 2 \dot{P}$ and the pulsar has not spun down much from its birth. If the magnetic field has the typical value of $10^{12.5} \mathrm{G}$, the current period is $100 \mathrm{msec}$, which is close to the initial

period. The problem with this hypothesis is that it fails by a factor $z 10^{2}$ in accounting for the energy content of the nebula. Either the methods used to estimate $\dot{E}$ are flawed or this pulsar has a very unusual magnetic field.

\section{Acknowledgements}

This work was supported in part by NSF grant AST-9016687.

\section{REFERENCES}

Aschenbach, B. \& Brinkmann, W. (1975). A\&A, 41, 147

Becker, R. H., Helfand, D. J., \& Szymkowiak, A. E. (1982). ApJ, 255, 557

Begelman, M. C. \& Li, Z.-Y. (1992). ApJ, 397, 187

Bietenholz, M. F. \& Kronberg, P. P. (1992). ApJ, 393, 206

Blandford, R. D., Applegate, J. H., \& Hernquist, L. (1983). MNRAS, 204, 1025.

Brown, G. E. \& Bethe, H. (1993), preprint

Brown, G. E., Bruenn, S. W., \& Wheeler, J. C. (1992). Comm. Ap., 16, 152

Caraveo, P. A., Bignami, G. F., Mereghetti, S., \& Mombelli, M. (1992). ApJ, 395, L103

Chevalier, R. A. (1977). Supernovae, ed. D. N. Schramm, Dordrecht, Reidel, p. 53 
Chevalier, R. A. (1989). ApJ, 346, 847

Chevalier, R. A. (1991). SN 1987 A and Other Supernovae, ed. I. J. Danziger, Garching, ESO, p. 511

Chevalier, R. A. \& Emmering, R. T. (1986). ApJ, 304, 140

Chevalier, R. A. \& Fransson, C. (1992). ApJ, 395, 540

Chevalier, R. A. \& Gull, T. R. (1975). ApJ, 200, 399

Colgate, S. A. (1971). ApJ, 163, 221

Emmering, R. T. \& Chevalier, R. A. (1989). ApJ, 345, 931

Fesen, R. A., Martin, C. L., \& Shull, J. M. (1992). ApJ, 399, 599

Frail, D. A. \& Moffett, D. A. (1993). ApJ, 408, 637

Gallant, Y. A., Hoshino, M., Langdon, A. B., Arons, J., \& Max, C. E. (1992). ApJ, 391, 73

Gunn, J. E. \& Ostriker, J. P. (1970). ApJ, 160, 979

Hoshino, M., Arons, J., Gallant, Y. A., \& Langdon, A. B. (1992). ApJ, 390, 454

Kaspi, V. M., Manchester, R. N., Johnston, S., Lyne, A. G., \& D'Amico, N. (1992). ApJ, 399, L155

Kennel, C. F. \& Coroniti, F. V. (1984a). ApJ, 283, 694

Kennel, C. F. \& Coroniti, F. V. (1984b). ApJ, 283, 710

Kirshner, R. P., Morse, J. A., Winkler, P. F., \& Blair, W. P. (1989). ApJ, 342, 260

Kulkarni, S. R., Predehl, P., Hasinger, G., \& Aschenbach, B. (1993). Nature, 362, 135

Li, Z.-Y. \& Begelman, M. C. (1992). ApJ, 400, 186

Manchester, R. N., Staveley-Smith, L., \& Kesteven, M. J. (1993). ApJ, 411, 756

Michel, F. C. (1988). Nature, 333, 644

Michel, F. C., Scowen, P. A., Dufour, R. J., \& Hester, J. J. (1991). ApJ, 368, 463

Murdin, P. \& Clark, D. H. (1981). Nature, 294, 543

Narayan, R. (1987). ApJ, 319, 162

Narayan, R. \& Schaudt, K. J. (1988). ApJ, 325, L43

Narayan, R. \& Ostriker, J. P. (1990). ApJ, 352, 222

Nomoto, K. \& Tsuruta, S. (1987). ApJ, 312, 711

Reynolds, S. P. (1985). ApJ, 291, 152

Reynolds, S. P. \& Fix, J. D. (1987). ApJ, 322, 673

Reynolds, S. P. \& Aller, H. D. (1988). ApJ, 327, 845

Seward, F. D., \& Wang, Z. (1988). ApJ, 332, 199

Seward, F. D., Harnden, F. R., Jr., Murdin, P., \& Clark, D. H. (1983). ApJ, 281, 650

Seward, F. D., Harnden, F. R., Jr., Szymkowiak, A., \& Swank, J. (1984). ApJ, 267, 698

Srinivasan, G., Bhattacharya, D., \& Dwarakanath, K. S. (1984). J. Ap. Astr., 5, 403

Stollman, G. M. (1987). A\&A, 178, 143

Suntzeff, N. B., Phillips, M. M., Elias, J. H., DePoy, D. L., \& Walker, A. R. (1992). ApJ, 384, L33

Trimble, V. L. (1968). AJ, 73, 535

Thorsett, S. E. (1992). Nature, 356, 690

van den Bergh, S. \& Pritchet, C. J. (1989). ApJ, 338, L69

van den Bergh, S. \& Tammann, G. (1991). ARA\&A, 29, 363

Velusamy, T. (1985). The Crab Nebula and Related Supernova Remnants, ed. M. C. Kafatos \& R. C. B. Henry, Cambridge, CUP, p. 115

Woosley, S. E., Pinto, P. A., \& Hartmann, D. (1989). ApJ, 346, 395 